\title{
Phase-shift amplification for precision measurements without nonclassical states
}

\author{
Alfredo Luis* \\ Departamento de Óptica, Facultad de Ciencias Físicas, Universidad Complutense, 28040 Madrid, Spain
}

(Received 18 March 2001; published 4 January 2002)

\begin{abstract}
We propose a practical arrangement that allows one to reach the Heisenberg limit in precision phase-shift measurements. This is achieved via phase-shift amplification. The arrangement we propose is based on experimental processes already carried out and does not require the use of any special quantum state.
\end{abstract}

DOI: $10.1103 /$ PhysRevA.65.025802

PACS number(s): 42.50.Dv, 03.65.-w

The quantum nature of physical processes limits how precisely some quantities can be measured. In this context, the detection of phase shifts is one of the most sensitive methods for determining very diverse physical magnitudes. Optimal phase-shift measurements are of importance to many areas, including precision spectroscopy and metrology, for instance. The quantum limits to phase-shift measurements are summarized by the so-called Heisenberg limit. By a variety of arguments it has been shown that the minimum detectable phase shift is of the order of the inverse of the number of particles involved in the measurement $[1,2]$.

Most approaches to the problem conclude that to reach the ultimate accuracy it is necessary to use quantum states with highly nonclassical properties [2]. In this work we show that this is not always the case. We demonstrate that the maximum precision can be achieved without using special quantum states. This is interesting because the use of nonclassical states imposes very stringent conditions to protect them against pernicious influences, such as decoherence, which can degrade their theoretical efficiency. The arrangement presented in this work allows to use classical or semiclassical states having a large number of particles (leading to an accordingly large resolution) without degrading the performance. The Heisenberg limit is reached via phase-shift amplification and employs currently available technology. Moreover, it is based on experimental processes already carried out successfully in the field of cavity quantum electrodynamics [3]. Similar schemes in the context of trapped ions can also be used for the precision phase-shift measurement presented in this work $[4,5]$.

We will consider a system consisting of a single bosonic degree of freedom (representing photons, phonons, or atoms, for example) described by the annihilation operator $a$, and a two-level system spanned by two orthogonal vectors $|e\rangle,|g\rangle$. For simplicity we assume that these are two internal electronic states of an atom.

The initial state $|\Psi\rangle$ undergoing the phase shift is the product of an arbitrary state $|\psi\rangle$ in mode $a$ and a 50\% superposition of $|g\rangle$ and $|e\rangle$,

$$
|\Psi\rangle=|\psi\rangle|\varphi\rangle,
$$

where

*Electronic address: alluis@eucmax.sim.ucm.es

$$
|\varphi\rangle=\frac{1}{\sqrt{2}}\left(|g\rangle+e^{i \phi_{0}}|e\rangle\right)
$$

and $\phi_{0}$ is a constant. The state in mode $a$ is assumed to be pure for simplicity. We would obtain exactly the same conclusions using mixed states.

The mechanism generating the phase shift is a dispersive coupling between the mode $a$ and the two-level atom governed by an interaction Hamiltonian of the form

$$
H \propto|e\rangle\langle e| a^{\dagger} a .
$$

This can be easily implemented in practice when $a$ is a field mode as well as when it represents the vibrational motion of a trapped ion $[3,5,6]$.

After a given interaction time, the evolution governed by the Hamiltonian (3) leads to the output state

$$
\begin{aligned}
\left|\Psi_{\phi}\right\rangle & =\exp \left[i \phi a^{\dagger} a|e\rangle\langle e|\right]|\Psi\rangle \\
& =\frac{1}{\sqrt{2}}\left(|\psi\rangle|g\rangle+e^{i \phi_{0}} e^{i \phi a^{\dagger} a}|\psi\rangle|e\rangle\right) .
\end{aligned}
$$

In order to infer the phase shift $\phi$ we consider a measurement performed on the two-level system described by projection on the states

$$
\left|\varphi_{ \pm}\right\rangle=\frac{1}{\sqrt{2}}(|g\rangle \pm|e\rangle)
$$

while no measurement is performed on the mode $a$. This measurement can be carried out by detecting the internal state of the atom $(|e\rangle$ or $|g\rangle)$ after applying a resonant laser pulse transforming $\left|\varphi_{ \pm}\right\rangle$into $|e\rangle,|g\rangle$ (i.e., a $\pi / 2$ pulse) [3]. There are only two possible outcomes $(+$ and -$)$ that appear with probabilities

$$
p_{ \pm}=\frac{1}{2}\left\{1 \pm \operatorname{Re}\left[e^{i \phi_{0}} C(\phi)\right]\right\},
$$

where

$$
C(\phi)=\left\langle\psi\left|e^{i \phi a^{\dagger} a}\right| \psi\right\rangle=\sum_{n=0}^{\infty} e^{i n \phi} \mathcal{P}(n)
$$


and $\mathcal{C}(\phi)$ and $\mathcal{P}(n)$ are the characteristic function and probability distribution, respectively, associated with the number operator $a^{\dagger} a$ in the state $|\psi\rangle$ [7].

The performance of this arrangement as a phase-shift detector depends on the function $\mathcal{C}(\phi)$ (which has been already used to study phase uncertainty in Ref. [8]). For simplicity, we assume a Gaussian form for $\mathcal{P}(n)$,

$$
\mathcal{P}(n) \propto \exp \left[-\frac{(n-\bar{n})^{2}}{2(\Delta n)^{2}}\right],
$$

where

$$
\begin{gathered}
\bar{n}=\left\langle\psi\left|a^{\dagger} a\right| \psi\right\rangle, \\
(\Delta n)^{2}=\left\langle\psi\left|\left(a^{\dagger} a\right)^{2}\right| \psi\right\rangle-\left\langle\psi\left|a^{\dagger} a\right| \psi\right\rangle^{2} .
\end{gathered}
$$

This particular $\mathcal{P}(n)$ is representative enough since it provides a useful approximation for many relevant states such as coherent and squeezed states of high intensity and moderate squeezing. (Nevertheless, for coherent and squeezed states there are simple exact expressions for $\mathcal{C}(\phi)$ [7].) When $\bar{n}$ and $\Delta n$ are large enough the sum in Eq. (7) can be safely replaced by an integral

$$
\begin{aligned}
C(\phi) & \simeq \frac{1}{\sqrt{2 \pi} \Delta n} \int_{-\infty}^{\infty} d n e^{i n \phi} \exp \left[\frac{(n-\bar{n})^{2}}{2(\Delta n)^{2}}\right] \\
& =e^{i \phi \bar{n}} e^{-(\Delta n)^{2} \phi^{2} / 2} .
\end{aligned}
$$

This leads to the following expression for the measured probabilities:

$$
p_{ \pm}=\frac{1}{2}\left[1 \pm e^{-(\Delta n)^{2} \phi^{2} / 2} \cos \left(\bar{n} \phi+\phi_{0}\right)\right] .
$$

The arrangement we are analyzing can be interpreted as an atomic interferometer of the Ramsey type, where $|g\rangle$ and $|e\rangle$ play the role of two interferometric paths that are recombined by atomic detection [3]. We can appreciate that the interference term depends on $\bar{n} \phi$ instead of the dependence on $\phi$ of standard interferometry. Therefore we can say that the phase shift $\phi$ has been amplified by a factor $\bar{n}$. The interference term is modulated by an exponential factor determining the visibility. This progressive lack of coherence depends on the number fluctuations $\Delta n$. Unit visibility is obtained provided that the phase shift is within some coherence range determined by the condition $\phi \ll 1 / \Delta n$. Actually this is not a restrictive condition since quantum limits are only meaningful for the detection of extremely minute phase shifts.

Next we examine the phase sensitivity of this scheme. We assume naturally that the phase shift to be detected is within the coherence range $(\phi \ll 1 / \Delta n)$ and we take $\phi_{0}=-\pi / 2$. In these conditions, the probabilities (6) become

$$
p_{ \pm} \simeq \frac{1}{2}[1 \pm \sin (\bar{n} \phi)] \text {. }
$$

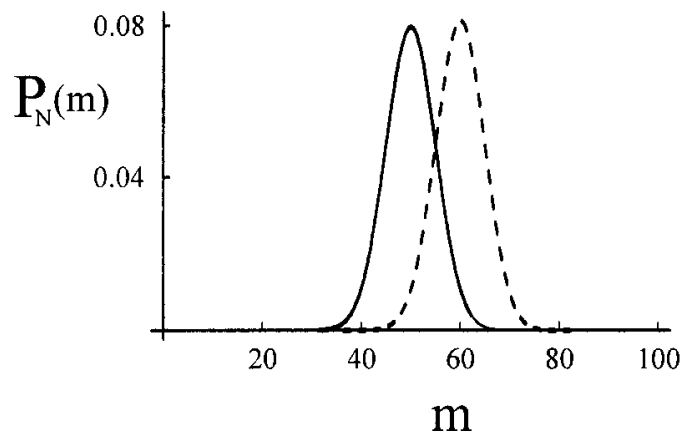

FIG. 1. Plot of the probability distribution $P_{N}(m)$ as a function of $m$ for $N=100$ and a coherent state with $\bar{n}=10^{9}$. We have represented $P_{N}(m)$ for two particular phase shifts: $\phi=0$ (solid line) and $\phi=2 \Delta \widetilde{\phi}=2 \times 10^{-10}$ (dashed line). No approximation has been made to obtain this plot.

Every single measurement has only two possible outcomes. Therefore, in order to obtain meaningful conclusions the measurement must be repeated several times. After $N$ repetitions, the probability that the outcome + is obtained $m$ times is given by the binomial distribution

$$
P_{N}(m)=\left(\begin{array}{c}
N \\
m
\end{array}\right) p_{+}^{m} p_{-}^{N-m}
$$

In the limit of large $N$ the quotient $m / N$ can be regarded as effectively continuous and the binomial distribution tends to be Gaussian,

$$
P_{N}(\widetilde{x}) \simeq \sqrt{\frac{N}{2 \pi}} \exp \left[-\frac{N}{2}(\tilde{x}-x)^{2}\right],
$$

where $\tilde{x}=2 m / N-1, x=\sin (\bar{n} \phi)$, and we have assumed that $x \ll 1$. We can appreciate that the measured variable $\tilde{x}$ is a suitable estimator of the true but unknown $x$. The uncertainty of this estimation can be expressed by the relation

$$
\Delta \widetilde{x}=\frac{1}{\sqrt{N}} .
$$

Since we are assuming that $x \ll 1$ we can consider the linearization $x \simeq \bar{n} \phi$ and $\widetilde{x} \simeq \bar{n} \widetilde{\phi}$, where $\widetilde{\phi}$ is the estimate of $\phi$. Then, Eq. (15) leads to

$$
\Delta \widetilde{\phi}=\frac{1}{\bar{n} \sqrt{N}} .
$$

The phase resolution scales as the inverse of the mean number of particles, so this measuring strategy approaches the Heisenberg limit.

For the sake of illustration we have represented in Fig. 1 the probability distribution (13) without any approximation using the exact probabilities (6) for a coherent state,

$$
p_{ \pm}=\frac{1}{2}\left[1 \pm e^{-2 \bar{n} \sin ^{2}(\phi / 2)} \cos \left(\bar{n} \sin \phi+\phi_{0}\right] .\right.
$$


We have used the parameters $\bar{n}=10^{9}, N=100$, and two phase shifts: $\phi=0$ (solid line) and $\phi=2 \Delta \widetilde{\phi}=2 \times 10^{-10}$ (dashed line). This plot confirms the correctness of the resolution (16). It can be easily seen that the plot in Fig. 1 does not depend appreciably on $\bar{n}$, so the accuracy can be arbitrarily increased by using a coherent state with $\bar{n}$ as large as possible.

In order to be precise, we recall that a phase-shift detection scheme reaches the Heisenberg limit when for a single measurement employing $\bar{n}$ particles, the sensitivity is $\Delta \widetilde{\phi}$ $=1 / \bar{n}$ (up to a multiplicative constant of the order of unity). This implies that when the measurement is repeated $N$ times and a maximum-likelihood analysis is used, the uncertainty is $\Delta \widetilde{\phi}=1 /(\bar{n} \sqrt{N})$, as in Eq. (16). This is the sense in which we say that the arrangement presented in this work reaches the Heisenberg limit for repeated measurements. Due to the lack of direct phase measurements, phase is always partially measured and partially inferred, and then the exact performance of the estimation depends on the data analysis used [9]. It must be also noted that the total number of particles used in a repeated measurement is $\bar{n} N$. Therefore, the arrangements reaching the Heisenberg limit should obtain a much better resolution if all the $\bar{n} N$ particles are used in a single measurement, at least in principle. Nevertheless, the actual quantum limits for multiple repeated measurements are not yet well known [10].

In this sense it is worth noting that, strictly speaking, the resolution (16) is only valid provided that we have a prior knowledge of $\phi$ with accuracy of the order of $1 / \bar{n}$ (this applies also to the proposals in Ref. [11], which rely also on phase-shift amplification). This is because of the combination of periodicity and amplification in Eqs. (11) and (12): for large $\bar{n} / \Delta n$ two phase shifts differing by $2 \pi / \bar{n}$ within the coherence range are indistinguishable since they lead to the same statistics $p_{ \pm}$. In the preceding calculations we have removed this ambiguity by assuming that $\phi$ was close enough to zero, so that Eq. (16) is fully meaningful. In most situations this a priori knowledge of $\phi$ can be obtained from the theory supporting the measurement. As a matter of fact a simple upper bound for $\phi$ can be enough. For example, it is expected that the pass of a gravitational wave would induce an interferometric phase shift of the order of $\phi \simeq 10^{-19}$ [12]. This situation parallels the free spectral range in spectroscopic measurements using Fabry-Perot interferometers or diffraction gratings [13]. In such an analogy $\bar{n}$ plays the role of the order of interference while $\sqrt{N}$ plays the role of the finesse (Fabry-Perot) or the number of slits (diffraction grating).

Phase-shift amplification can be achieved also by using maximally entangled states $[2,11]$. These states are highly nonclassical and can be regarded as examples of Schrödinger cats: a coherent superposition of distinct states [14]. It is worth stressing that in our case no entanglement is required. The amplification can be obtained for a very large class of states without special quantum properties. For instance, coherent states can be used. We may say that in our case the quantum nature is put to the atom interacting with the light field (in the form of a coherent superposition of $|e\rangle$ and $|g\rangle$ ) instead of the light itself. We think that this is advantageous because these atomic superpositions are nowadays efficiently produced in the laboratory, in contrast to special quantum states of light such as maximally entangled states. Moreover, this allows to increase arbitrarily the number $\bar{n}$ of particles without the degrading effects caused by the decoherence that strongly affects the nonclassical states of light.

Furthermore, we obtain exactly the same results if the initial state is mixed instead of pure. For example, for a coherent state it can be seen in Eq. (17) that the statistics do not depend on the phase of the corresponding complex amplitude. This means that a phase-averaged coherent state reaches exactly the same sensitivity. This further simplifies the practical implementation of this scheme. We can also exclude the generation of Schrödinger cats during the process. On the contrary, the states $|\psi\rangle$ and $e^{i \phi a^{\dagger} a}|\psi\rangle$ in Eq. (4) must not be distinct if interference with meaningful visibility is desired. Visibility close to 1 implies that $\phi \ll 1 / \Delta n$, so from Eq. (10) we have that $\left|\left\langle\psi\left|e^{i \phi a^{\dagger} a}\right| \psi\right\rangle\right| \simeq 1$.

We stress that this measuring arrangement can be implemented in practice since it is based on processes already proposed and carried out experimentally. As a matter of fact, the probabilities (11) have been already obtained in the fields of cavity quantum electrodynamics [3] and trapped ions [4,5] when studying the generation of Schrödinger cats.

Finally, we note that this phase-shift amplification is different from the phase-amplification concept contained in Ref. [15]. In our case we have the effective amplification of a classical parameter, the phase shift. This must not be confused with the amplification of quantum variables, such as the quantum phase as it has been studied in Ref. [15]. In particular, it can be noted that this parameter amplification does not require any additional physical process or external energy supply. This is purely an effect of the measuring strategy adopted leading to the statistics of the form (6). All this means that the phase-shift amplification is not constrained by the conditions that quantum mechanics (mainly the commutation relations) imposes on the amplification of quantum variables, usually leading to the addition of noise [16]. Among the differences that this fact introduces we have that the amplification of the quantum phase variable is independent of the state of the system, at the expense of unavoidably introducing additional noise, while the phase-shift amplification discussed here depends on the state of the system, but no fluctuations are added.

Summarizing, we have proposed a practical measuring arrangement for reaching the Heisenberg limit, which is based on currently available experimental processes. The statistics of the proposed measurement reveals that there is phase amplification between the application of the phase shift and its detection. In contrast to other schemes, in this case the quantum limit is reached without employing any kind of special quantum states. This is interesting since it allows one to use states with very large numbers of particles without caring about decoherence effects that are so pernicious for special quantum states such as maximally entangled states or Schrödinger cats. 
[1] Z. Y. Ou, Phys. Rev. A 55, 2598 (1997).

[2] A. Luis and L. L. Sánchez-Soto, Progress in Optics, edited by E. Wolf (Elsevier, Amsterdam, 2000), Vol. 41, p. 421.

[3] M. Brune, S. Haroche, J. M. Raimond, L. Davidovich, and N. Zagury, Phys. Rev. A 45, 5193 (1992); L. Davidovich, A. Maali, M. Brune, J. M. Raimond, and S. Haroche, Phys. Rev. Lett. 71, 2360 (1993); M. Brune, E. Hagley, J. Dreyer, X. Maitre, A. Maali, C. Wunderlich, J. M. Raimond, and S. Haroche, ibid. 77, 4887 (1996); L. Davidovich, M. Brune, J. M. Raimond, and S. Haroche, Phys. Rev. A 53, 1295 (1996); J. M. Raimond, M. Brune, and S. Haroche, Phys. Rev. Lett. 79, 1964 (1997); S. Haroche, Phys. Today 51 (7), 36 (1998).

[4] C. Monroe, D. M. Meekhof, B. E. King, and D. J. Wineland, Science 272, 1131 (1996).

[5] J. C. Retamal and N. Zagury, Phys. Rev. A 55, 2387 (1997); C. C. Gerry, ibid. 55, 2478 (1997).

[6] C. M. Savage, S. L. Braunstein, and D. F. Walls, Opt. Lett. 15, 628 (1990); W. Vogel and R. L. de Matos Filho, Phys. Rev. A 52, 4214 (1995); R. L. de Matos Filho and W. Vogel, ibid. 58, R1661 (1998).

[7] J. Peřina, Quantum Statistics of Linear and Nonlinear Optical Phenomena (Kluwer, Dordrecht, 1991).

[8] M. Hillery, M. Freyberger, and W. Schleich, Phys. Rev. A 51, 1792 (1995).
[9] J. Peřina, Z. Hradil, and B. Jurčo, Quantum Optics and Fundamentals of Physics (Kluwer Academic, Dordrecht, 1994); M. Zawisky, Y. Hasegawa, H. Rauch, Z. Hradil, R. Myška, and J. Peřina, J. Phys. A 31, 551 (1998).

[10] S. L. Braunstein, A. S. Lane, and C. M. Caves, Phys. Rev. Lett. 69, 2153 (1992); A. S. Lane, S. L. Braunstein, and C. M. Caves, Phys. Rev. A 47, 1667 (1993).

[11] J. Jacobson, G. Björk, I. Chuang, and Y. Yamamoto, Phys. Rev. Lett. 74, 4835 (1995); J. J. Bollinger, W. M. Itano, D. J. Wineland, and D. J. Heinzen, Phys. Rev. A 54, R4649 (1996); S. F. Huelga, C. Macchiavello, T. Pellizzari, A. K. Ekert, M. B. Plenio, and J. I. Cirac, Phys. Rev. Lett. 79, 3865 (1997); V. Meyer, M. A. Rowe, D. Kielpinski, C. A. Sackett, W. M. Itano, C. Monroe, and D. J. Wineland, ibid. 86, 5870 (2001).

[12] D. F. Walls and G. J. Milburn, Quantum Optics (SpringerVerlag, Berlin, 1994).

[13] R. S. Longhurst, Geometrical and Physical Optics (Longmans, London, 1967).

[14] N. D. Mermin, Phys. Rev. Lett. 65, 1838 (1990).

[15] A. Luis and L. L. Sánchez-Soto, J. Phys. A 24, 2083 (1991); Quantum Opt. 5, 33 (1993); Phys. Rev. A 47, 1492 (1993); G. M. D’Ariano, C. Macchiavello, N. Sterpi, and H. P. Yuen, ibid. 54, 4712 (1996).

[16] C. M. Caves, Phys. Rev. D 26, 1817 (1982). 